\title{
PERFIL SOCIODEMOGRÁFICO E NARRATIVAS DE PESSOAS IDOSAS QUE JÁ VIVIAM A SOLIDÃO ANTES DA SINDEMIA DO COVID-19 1
}

Sociodemographic profile and narratives of older adults those who already lived in loneliness before the covid 19 syndemic

BEZERRA, Patrícia Araújo ${ }^{2}$

SOARES, Suelen de Alencar ${ }^{3}$

FRANCO, Simone Bezerra 4

MOURA, Leides Barroso de Azevedo ${ }^{5}$

\begin{abstract}
Resumo: Os dados e narrativas apresentados neste estudo descrevem o quão urgente é compreender os desafios e as oportunidades da heterogeneidade do envelhecimento, especialmente numa sociedade ageista que historicamente isola os indivíduos mais longevos. Neste artigo são analisados resultados de entrevistas com pessoas de 60 anos ou mais de idade residentes em duas Instituições de Longa Permanência no Distrito Federal, no período de agosto a dezembro de 2019. Adota-se o constructo do ageismo como perspectiva teórica do estudo. Observou-se que a maioria das pessoas idosas já viviam a solidão antes da circulação global do SARS COV-2. A sindemia do COVID 19 em curso tem o potencial de acirrar a solidão já vivida por pessoas idosas, mas também de criar novas sociabilidades e redes de solidariedade intergeracionais.
\end{abstract}

Palavras-chave: Solidão. Sindemia. Covid-19. Pessoa Idosa. Ageismo.

\begin{abstract}
The data and narratives presented in this study describe how urgent is to understand the challenges and opportunities of the heterogeneity of aging, especially in an age-based society that historically isolates the most long-lived individuals. This article analyzes the results of interviews with people aged 60 or over who live in two Long-Term Institutions in the Federal District in Brazil, from August to December 2019. The ageism construct is adopted as the theoretical perspective of the study. It was observed that the majority of older adults already lived in loneliness, even before the global circulation of SARS COV-2. The current COVID 19 syndemic has the potential to intensify the social isolation and perception of loneliness already experienced by older persons, but it also has the potential to create new forms of sociability and intergenerational solidarity networks.
\end{abstract}

Keywords: Loneliness. Syndemic. Covid-19. Elderly. Ageism.

\footnotetext{
${ }^{1}$ Recebido em: 03 Nov. 2020 | Aceito em: 05 Dez. 2020.

2 Doutoranda em Desenvolvimento, Sociedade e Cooperação Internacional, UnB, Brasil. Professora do Departamento de Enfermagem do Centro Universitário do Distrito Federal, UDF, Brasil pat.araujobezerra@gmail.com.

${ }^{3}$ Graduanda de Enfermagem da Universidade de Brasília, Unb, Brasil. suelenalencarsoares@gmail.com

4 Doutoranda em Desenvolvimento, Sociedade e Cooperação Internacional, UnB, Brasil. simone.bezerrafranco@gmail.com

5 Professora Associada do Departamento de Enfermagem e do Programa de Pós-Graduação em Desenvolvimento, Sociedade e Cooperação Internacional da Universidade de Brasília, UnB, Brasil. leidesm74@gmail.com
} 


\section{Introdução}

De acordo com o Instituto Brasileiro de Geografia e Estatística (IBGE, 2018) no ano de 2010 havia cerca de 19, 3 milhões de pessoas com 60 anos ou mais, representando 10,3\% da população total. Atualmente existem cerca de 28 milhões de brasileiros idosos e, em 2050, esse quantitativo chegará a 66,4 milhões de pessoas, representando 29,3\% da população total. Na América Latina, os países em desenvolvimento que possuem uma economia periférica, como é o caso do Brasil, vivenciam processos de envelhecimento acelerado e, apesar das desigualdades, o envelhecimento deve ser considerado uma conquista do desenvolvimento, como processo inevitável. 0 país ocupa a quinta posição global de maior população de idosos (Lima-Costa, 2018).

Entretanto, viver mais não significa viver com maior qualidade de vida. 0 envelhecimento populacional tem ocorrido em um contexto de transformações estruturais acentuadas nas famílias, avanços tecnológicos, mudanças na nupcialidade, queda da fecundidade, ingresso maciço das mulheres no mercado de trabalho, aumento dos fenômenos de individualização e diversidades de condições sociais, econômicas, culturais e ambientais. (Moura et al, 2019; Veras \& Oliveira, 2018; Camarano et al, 2010; Elliott \& Lemert, 2006).

Neste caminho, a solidão tem se destacado no campo da saúde como um dos maiores problemas potenciais de saúde mental, sobretudo quando relacionada à população idosa (Park et al, 2018; Tadaka, 2016) e no contexto atual de sindemia, que representa a pandemia do COVID 19 na presença de desigualdades e iniquidades sociais (Horton, 2020).

Esta realidade já era apresentada antes da pandemia do COVID-19 e, está diretamente relacionada ao que Robert Butler (1980) apresenta como ageismo, que configura um conjunto de estereótipos, antipatias, fugas de contato, embasadas em mitos que de forma sistemática geram discriminação contra pessoas idosas e produzem preconceitos historicamente construídos na sociedade moderna, que ampliam barreiras de interação e inserção social das mais diversas ordens e dificultam a compreensão do envelhecimento como processo, colocando-o como um problema social. Ainda nesta 
questão, Beauvoir (1970) já apontava, que sob o ângulo da produção e do lucro, as narrativas e a organização sociocultural oprimem a velhice, diminuindo possibilidades de desfrutar da vida longa.

Outra perspectiva advém de Guita Debert (1997), que considera a terceira idade como uma invenção, fruto de um processo crescente de socialização da gestão da velhice e que significou transformá-la de uma experiência privada e familiar para uma questão pública. Essa invenção gerou como consequência sucessivas tentativas de homogeneização da velhice pelo aparelho estatal, para intervenções, abrindo mercados de consumos e diminuindo singularidades, despotencializando pessoas mais velhas e comprometendo a capacidade da sociedade de funcionar sobre bases de reconhecimento mútuo e solidariedade intergeracional. A condição subalternizada (Spivac, 2010) da pessoa idosa pode colocá-la em risco de exclusão da participação social.

Este contexto, portanto, já propiciava antes da Pandemia pelo COVID-19, um aumento sem precedentes da vida solitária e representa, portanto, uma das mudanças sociais mais significativas do mundo moderno (Tadaka, 2016).

Como o surgimento do coronavírus em 2019 no mundo, um "novo normal" passou a existir e uma das formas de combate a disseminação do vírus foi o distanciamento social. A emergência sanitária que colocou milhões de pessoas em condições de distanciamento, diminuiu o número de interações sociais e ampliou sentimentos e percepções de solidão, porém, esta realidade já existia na subjetividade e vivência de pessoas idosas

Esta realidade anterior é fruto da interação de sistemas de opressão, de exclusão, do ageismo, assim como o racismo, o sexismo, o machismo, entre outros. 0 ageismo também pode ser discutido sob o paradigma do produtivismo, no qual as demandas no neoliberalismo obscurecem narrativas, histórias de vidas das pessoas idosas, diminuindo as chances de redes de solidariedade, apoio e vida digna (Moura, 2019; OMS, 2008).

Diante disto, o presente artigo possui como objetivo descrever a percepção de solidão vivenciada por pessoas idosas residentes em duas Instituições de Longa Permanência do Distrito Federal antes do contexto pandêmico e, refletir as consequências e aproximações daquela realidade com o confinamento atual. 


\section{Desenvolvimento}

\section{Método}

Trata-se de um estudo de abordagem mista, do tipo transversal e de natureza analítica. Realizou-se coleta de dados por meio de entrevistas com pessoas idosas residentes em duas Instituições de Longa Permanência. Utilizou-se instrumento do tipo sistema fechado por intermédio da Escala Brasileira de Solidão - UCLA Loneliness. A coleta de dados ocorreu de agosto a dezembro de 2019.

Para aplicação do instrumento foram convidados 29 idosos, com capacidades cognitivas preservadas, que residem em Instituições de Longa Permanência (ILP) no Distrito Federal, uma pública e outra conveniada ao Sistema de Assistência Social e se constituem como locais de moradia coletiva para pessoas idosas, direcionadas ao acolhimento e assistência especializada em gerontologia e geriatria. Por questões éticas são preservados os nomes e as características das ILPI.

A Escala de Solidão UCLA Revisada (R-UCLA), desenvolvida por Russel et al (1978) tem sido usada com frequência em vários países em pesquisas envolvendo grupos etários, em especial pessoas idosas, segundo estudo do Universidade Federal do Triângulo Mineiro (UFTM) e Departamento de Psicologia (2016). A escala UCLA apresenta boas qualidades psicométricas, indicando capacidade para avaliar a solidão de respondentes entre 20 e 87 anos e no contexto brasileiro, apresentou bom índice de fidedignidade, indicando ser uma medida confiável, de validade de construto e discriminante (Barroso et al, 2016).

Essa versão revisada da Escala de Solidão UCLA contém 20 questões do tipo afirmativas com respostas no sistema likert de quatro pontos, para alternativas que descrevem as interações sociais da pessoa idosa. Sobre suas propriedades psicométricas em estudo anterior validado no Brasil a escala apresentou boa consistência interna $(\alpha=$ $0,94)$, correlação com a autopercepção sobre a solidão $(r=0,70)$ e validade discriminativa 
com diversos outros conceitos, entre eles a depressão $(r=0,50)$, autoestima $(r=-0,49)$, introversão/extroversão $(r=-0,46)$, tendência à afiliação $(r=-0,45)$, ansiedade $(r=0,36)$, assertividade $(r=-0,34)$, sensibilidade à rejeição $(r=0,27)$ e desejabilidade social $(r=-$ $0,20)$. A solidão é avaliada como mais intensa à medida que a pontuação é maior na soma total das respostas aos itens (Barroso et al, p.70, 2016).

A análise apresentada nos resultados está organizada em três categorias: 1) perfil sociodemoráfico da população estudada, onde são detalhadas as características como renda, escolaridade, raça/cor, entre outros; 2) análise da autopercepção de solidão, onde são trazidos alguns resultados que evidenciam características do grupo analisado e 3) relatos curtos das memórias das pessoas idosas, captados no momento de entrevista.

Os riscos da pesquisa foram mínimos e todas as pessoas idosas que participaram voluntariamente da pesquisa assinaram o Termo de Consentimento Livre e Esclarecido (TCLE), conforme Resolução do Conselho Nacional de Saúde CNS n. 466/2012. O protocolo de pesquisa foi aprovado pelo Comitê de Ética em Pesquisa da Faculdade de Saúde, da Universidade de Brasília, sob o número CAAE: 14105119.0.0000.0030.

\section{Resultados e Discussão}

\section{Perfil Sociodemográfico}

O perfil sociodemográfico revelou que das 29 pessoas idosas entrevistadas a maioria é composta por mulheres, pardas/pretas, de escolaridade até o ensino fundamental, católica, solteira e com renda de até um salário-mínimo.

As mulheres representam um total de 16 indivíduos (55,2\%) e os homens 13 (44,8\%), com uma mediana de idade de 76 anos, sendo a pessoa idosa mais jovem com 62 anos e o mais velho com 96 anos de idade. Do total dos entrevistados, 13 pessoas (44\%) possuíam mais de 80 anos. Quanto a cor auto-declarada, 16 (55,2\%) pessoas se declararam parda/preta, 12 (41,4\%) brancas e 1 (3,4\%) amarela/indígena. Em relação à escolaridade, 16 (55,2\%) possuem ensino fundamental, 7 (24,1\%) ensino médio e, 
apenas, $3(10,3 \%)$ ensino superior. No aspecto religioso, $19(65,5 \%)$ são católicos, 6 (20,7\%) evangélicos, 2 (6,9\%) espíritas e 2 (6,9\%) não possuem religião.

Em relação ao detalhamento do estado civil, 12 (41,4\%) afirmaram estarem solteiros, $7(24,1 \%)$ viúvos, $4(13,8 \%)$ divorciados, 4 (13,8\%) separados e 2 (6,8\%) casados. Dentre os entrevistados 11 (41,3 \%) possuem renda de até um salário-mínimo, $7(24,1 \%)$ não souberam informar ou precisar um valor, $5(17,2 \%)$ recebem de 01 a 03 salários-mínimos, $4(13,7 \%)$ não possuem renda e apenas $1(3,4 \%)$ possui renda acima de 04 salários-mínimos.

\section{Análise de Autopercepção de Solidão - Escala UCLA-Loneliness}

A análise realizada nesta etapa procura apresentar os dados mais gerais e que foram mais marcantes no resultado da entrevista com a escala UCLA-Loneliness.

Sobre dimensões da solidão $12(41,4 \%)$ das pessoas idosas entrevistadas apontaram não se sentirem frequentemente próximos a ninguém. A mesma proporção de idosos entrevistados afirmou que suas ideias e interesses não são compartilhados por aqueles que os rodeiam. Um outro dado que chamou atenção, está relacionado com as aproximações das relações. Um total de 16 (55,2\%) pessoas revelaram que suas relações sociais são na maior parte das vezes superficiais. Além disto, 11 (37,9\%) pessoas apontaram que frequentemente sente-se carentes de companhia. Por fim, 34,5\% sentem que as pessoas estão ao redor deles, mas não estão com eles e, por fim, sete indivíduos revelaram sentir-se completamente sozinhos $(24,1 \%)$.

Alguns dados estão retratados nos gráficos (Gráfico 1; Gráfico 2; Gráfico 3) a seguir. 


\section{Gráfico 1}

Resultado da pergunta 3 da Escala UCLA para os 29 idosos pesquisados nas ILP, 2019. "Eu sinto que não tenho companhia".

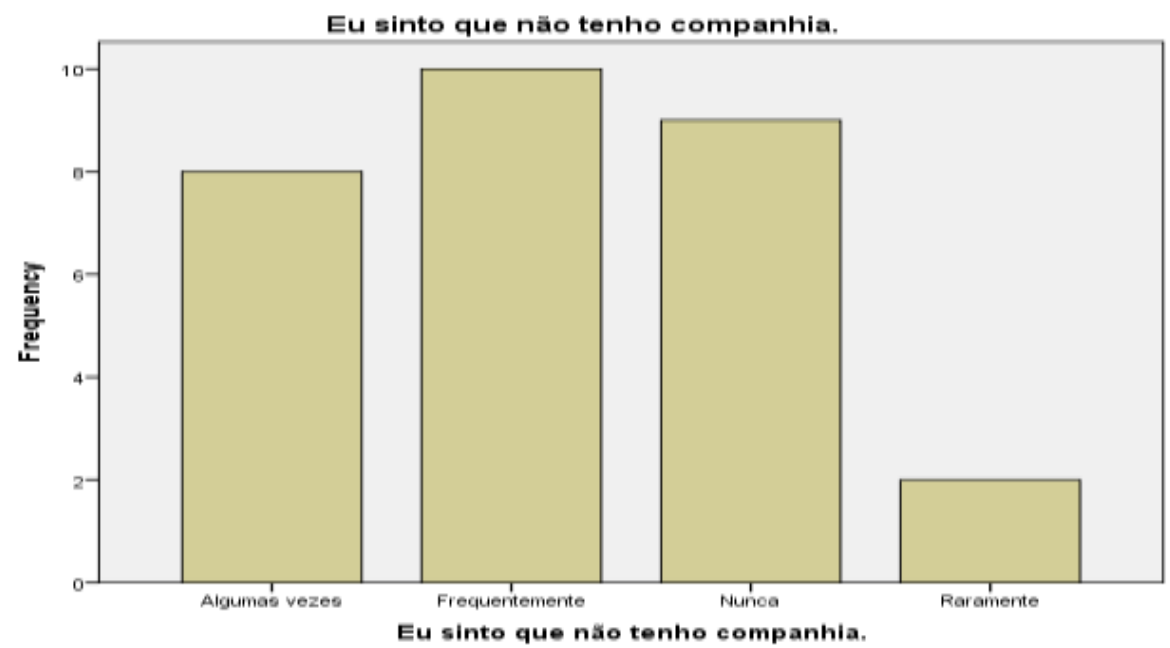

\section{Gráfico 2}

Resultado da pergunta 7 da Escala UCLA para os 29 idosos pesquisados nas ILP, 2019. "Eu não me sinto próximo (a) a ninguém".

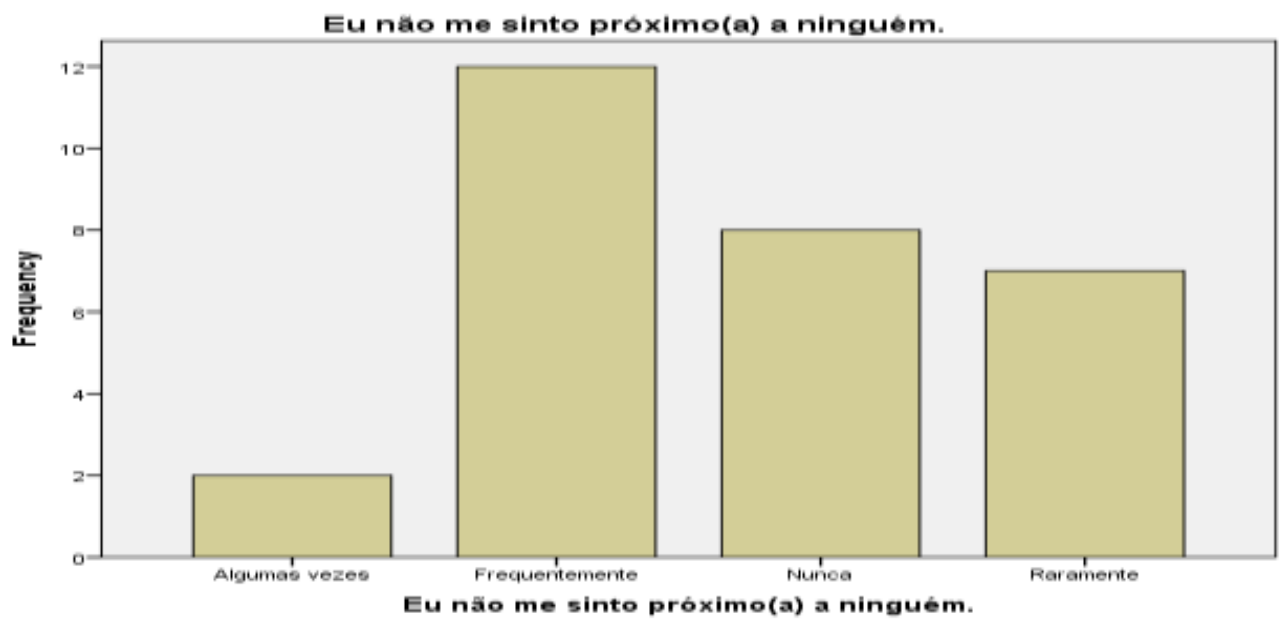




\section{Gráfico 3}

Resultado da pergunta 10 da Escala UCLA para os 29 idosos pesquisados nas ILP, 2019. "Eu me sinto completamente sozinho (a)".

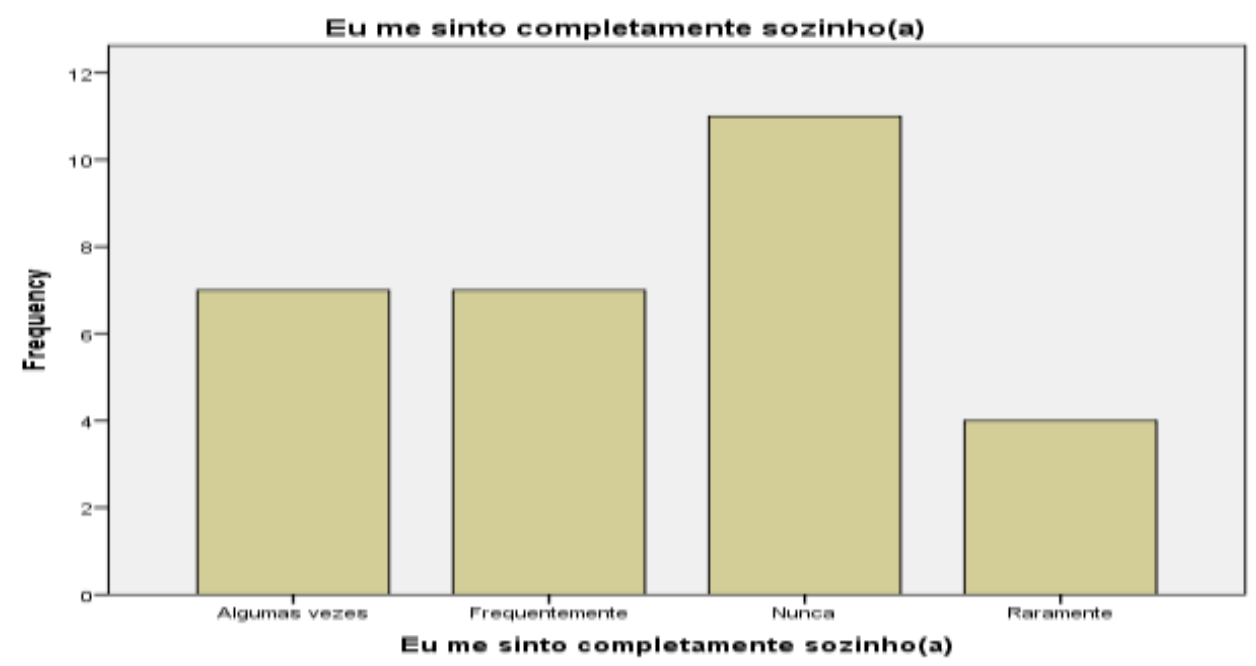

\section{Breves relatos captados no momento da coleta de dados que revelam subjetividades da experiência da solidão}

Durante o processo da entrevista foi possível perceber e anotar, como um diário de campo, algumas narrativas das pessoas idosas, que estão apresentadas a seguir.

"Aqui não tem graça nenhuma viver". S.U.E.U., 93 anos.

"Gostaria de ter uma vida mais produtiva, gostaria de trabalhar. Sou cubana e me sinto excluída”. T.A.S., 66 anos.

"Eu me sinto recluso aqui. O lugar é bom, mas a convivência é limitada. Tem idosos de idade bastante avançada e não consigo me comunicar com nenhum". O.P.S., 72 anos.

"Eu vivo uma vida muito isolada. Meus filhos moram no Rio, mas tenho uma filha que mora em Brasília. Aqui não tenho com quem dialogar, como engenheiro eu gostaria de conversar coisas técnicas." H.A.F., 86 anos. 
"Não tenho ninguém aqui que corresponda a minha necessidade de discutir no meu nível de educação". A.M.G.R.S., 73 anos.

"Tem uma idade que as pessoas não entendem". (Dificuldade em fazer amigos) "Hoje em dia porque estou muito velha e as pessoas velhas têm muitos poucos amigos. Eu me considero uma pessoa só, pois não tenho família, apenas poucos amigos". M.M.S, 96 anos.

"As pessoas não sabem me entender. Não tenho amizades, me entende?" M.F.C.S, 65 anos.

“Eu não tolero ficar tão sozinho" (...) “Já me acostumei”. J.I.P, 71 anos.

Os relatos, espontaneamente apresentados revelam dimensões de percepções de solidão e desvelam o que Wong et al (2017) apontam como uma solidão ética relacionada à experiência de alienação de pessoas idosas no nível social que são influenciadas por, entre outras coisas, abandono. Ou ainda, ao que Park (2018) apresenta como sendo o baixo nível de engajamento em redes familiares e de amigos que gera inúmero reflexos, entre eles redução do bem-estar, sensação de vida insatisfatória (Ramage-Morin, 2016) e aumento da morbimortalidade (Tadaka, 2016).

Tais achados aproximam o debate da situação atual da pandemia pelo COVID-19, quando se evidencia que o distanciamento social já era algo experimentado por outras pessoas. Claramente por causas diferentes, a aproximação com a experiencia subjetiva vivida pelas pessoas idosas isoladas e solitárias daqueles milhões e milhões de pessoas se viram abrigados a permanecerem em suas residências confinados e a diminuírem os contatos sociais, contraditoriamente, evidencia a marginalização a que estas pessoas idosas sempre estiveram submetidas, em um contexto de naturalização das suas diferenças.

Igualmente, não são todos os grupos sociais que realizaram a quarentena, como afirma Oliveira (2020), porque usualmente os grupos periféricos tiveram que sair para trabalhar, mas, o contraste aqui apresentado pelos idosos institucionalizados é que se trata de um grupo que já se percebia anteriormente excluído e que podem continuar nessa condição de subalternizado (Spivac, 2010) e invisibilizados. As condições singulares de 
vida de idosos institucionalizados podem estar mais acirradas em detrimento da sindemia e representar um problema social global.

\section{Considerações Finais}

Pessoas idosas institucionalizadas já percebiam situações de solidão prepandemia. No limite, o que se questiona para o futuro é como estas experiências poderão ressignificar o cuidado de pessoas idosas residentes em ILPI? Poderão os idosos, a partir da vivência de confinamento, reconfigurar os laços sociais e encontrar uma solidariedade intergeracional com grupos de outras faixas etárias? Ou as condições de desigualdade serão acirradas, colocando para um confinamento ainda maior a população idosa que, muito em breve, será a maior parte da sociedade mundial?

0 artigo procurou evidenciar e registrar a realidade daqueles que já viviam em solidão, fruto de um processo estruturante de limitação de oportunidades de cuidado nos locais onde se viveu. 0 estudo aponta quão urgente é compreender os desafios e oportunidades deste momento que o mundo está vivendo de rápido envelhecimento e no contexto de cuidado em ILPI.

Entre as lutas de resistência advindas do movimento negro, de mulheres, das populações lgbt's, entre outras, deve-se agregar a daqueles que possuem o dom de contar histórias, lembrar memórias e unir o passado com o presente. Talvez, a experiência subjetiva e coletiva de distanciamento social, possa representar uma esperança para ressignificação social e reconstrução de laços mais solidários e intergeracionais para idosos institucionalizados. 


\section{Referências}

BARROSO, S.M. et al. Evidências de validade da Escala Brasileira de Solidão UCLA. Uberaba - MG: Universidade Federal do Triângulo Mineiro (UFTM), Departamento de Psicologia, 2016.

BEAUVOIR, S. A Velhice. Rio de Janeiro: Nova Fronteira, 1990.

BRASIL. Ministério do Planejamento, Orçamento e Gestão. Instituto Brasileiro de Geografia e Estatística. Diretoria de Pesquisas. Coordenação de População e Indicadores Sociais. Projeções da População do Brasil e Unidades da Federação por sexo e idade: 2010 2060. Rio de Janeiro: Instituto Brasileiro de Geografia e Estatística; 2018.

BUTLER, R. N. Ageism: A foreword. Journal of Social Issues, v. 36, n. 2, p. 8-11, 1980.

CAMARANO, A.M; KANSO, S. As instituições de longa permanência para idosos no Brasil. Rev. bras. Estud. Popul. vol.27 no.1 São Paulo Jan./June 2010.

DEBERT, G. G. A invenção da terceira idade e a rearticulação de formas de consumo e demandas políticas. Revista Brasileira de Ciências Sociais, v. 12, n. 34, p. 39-56, 1997.

ELLIOTT, A.; LEMERT, C. The New Individualism - The Emotional Costs of Globalization. Londres e Nova Iorque: Routledge, 2006.

HORTON, R. COVID 19 is not a pandemic. The Lancet, v. 396, n. 10255, p 874, 2020.

LIMA-COSTA, M. F., 2018. Envelhecimento e Saúde coletiva: Estudo Longitudinal da Saúde dos Idosos Brasileiros (ELSI-Brasil). Rev Saúde Pública. 52 Supl 2:2s.

MOURA, L B A. A Pessoa Idosa na Área Metropolitana de Brasília: oportunidades e desafios. In: Vasconcelos A M N et all (Org) Território e sociedade: as múltiplas faces da Brasília metropolitana. Brasília: Editora UnB; 2019.

OLIVEIRA, A. A quarentena é branca / classe, raça, gênero e colonialidade. Ver. REALIS, v. 10, n. 01, p. 1903-203, 2020.

ORGANIZAÇÃO MUNDIAL DE SAÚDE. Guia Global das Cidades Amigas da Pessoa Idosa. Genebra: OMS; 2008.

PARK, N. S. et al. Associations of a social network typology with physical and mental health risks among older adults in South Korea. Aging \& mental health, v. 22, n. 5, p. 631-638, 2018.

RAMAGE-MORIN, P.L. Hearing difficulties and feelings of social isolation among Canadians aged 45 or older. Statistics Canada, 2016. 
RUSSELL D, PEPLAU LA, FERGUSON ML. Developing a measure of loneliness. J Pers Assess. 1978;42(3):290-4.

SPIVAC G.C. Pode o subalterno falar? Belo Horizonte: editora UFMG; 2010

TADAKA, E. et al. Development of a community's self-efficacy scale for preventing social isolation among community-dwelling older people (Mimamori Scale). BMC public health, v. 16, n. 1, p. 1198, 2016.

VERAS, R. P., \& OLIVEIRA, M. (2018). Envelhecer no Brasil: a construção de um modelo de cuidado. Ciência \& Saúde Coletiva, 23, 1929-1936. 